\title{
Intervenientes dos processos de transferência tecnológica em uma instituição de ciência e tecnologia: o caso FIOCRUZ
}

Fernando Victor Cavalcante,

Mestrando do Programa de Pós-Graduação em Gestão e Estratégia (MPGE) da Universidade Federal Rural do Rio de Janeiro

fervicante@yahoo.com.br

Monique Brandão Comes de Almeida

Mestranda do Programa de Pós-Graduação em Gestão e Estratégia (MPGE) da Universidade Federal Rural do Rio de Janeiro

monique.comes@gmail.com

Thiago Borges Renault

Doutor em Engenharia de Produção pela Universidade Federal do Rio de Janeiro . Professor do Departamento de Ciências Administrativas e Contábeis (DCAC) e Professor Permanente do Programa de Pós-Graduação em Gestão e Estratégia (MPGE) da Universidade Federal Rural do Rio de Janeiro thiagorenault@gmail.com

Editor Científico: José Edson Lara

Organização Comitê Científico

Double Blind Review pelo SER/OJS

Recebido em 30.04.2018

Aprovado em 12.07.2018 


\title{
Resumo
}

Esta pesquisa objetiva investigar os intervenientes dos processos de transferência tecnológica da Fiocruz, a partir da perspectiva dos gestores do sistema de gestão tecnológica institucional. Para o desenvolvimento da pesquisa foi adotado o Estudo de Caso único com evidências coletadas por meio de entrevistas semiestruturadas, pesquisa documental e observação. Identificaram-se como motivadores primários o reconhecimento científico e o cumprimento da função social da instituição. Entre as principais barreiras estão: cultura não orientada para a transferência de tecnologia, morosidade do processo e ausência de habilidades de valoração e negociação. Já entre os facilitadores, destacam-se: infraestrutura institucional, imagem institucional e competências relativas à propriedade intelectual. Os resultados, além de identificar a existência de intervenientes, permitiram corroborar estudos anteriores e criar, a partir de dados empíricos, uma taxonomia de intervenientes, apresentando sua principal contribuição aos estudos sobre transferência de tecnologia.

Palavras-chave: Escritórios de Transferência de Tecnologia; Intervenientes em Processos de Transferência Tecnológica; Transferência de Tecnologia.

\section{Intervening factors in the technological transfer in a science and technology institution: the case of FIOCRUZ}

\begin{abstract}
This research aims to investigate the intervening factors in the Fiocruz technology transfer processes, from the perspective of the managers of the institutional technological management system. For the development of the research was adopted the single Case Study with evidence collected through semi-structured interviews, documentary research and observation. Scientific recognition and fulfillment of the institution's social function were identified as primary motivators. The main barriers are: culture not oriented to technology transfer, slowness of the process and lack of valuation and negotiation skills. Among the facilitators, the following stand out: institutional infrastructure, institutional image and competences related to intellectual property. The results, besides identifying the existence of intervening factors, allowed to corroborate previous studies and to create a taxonomy of intervening factors based on empirical data, presenting their main contribution to studies on technology transfer.
\end{abstract}

Keywords: Technology Transfer Offices; Intervening Factors in the Technological Transfer Processes; Technology Transfer.

\section{Intervinientes de los procesos de transferencia tecnológica en una institución de ciencia y tecnología: el caso FIOCRUZ}

\section{Resumen}

Esta investigación objetiva investigar los intervinientes de los procesos de transferencia tecnológica de Fiocruz, desde la perspectiva de los gestores del sistema de gestión tecnológica institucional. Para el desarrollo de la investigación fue adoptado el Estudio de Caso único con evidencias recolectadas por entrevistas semiestructuradas, investigación documental y 
observación. Se han identificado como motivadores primarios el reconocimiento científico y el cumplimiento de la función social de la institución. Entre las principales barreras están: cultura no orientada a la transferencia de tecnología, morosidad del proceso y ausencia de habilidades de valoración y negociación. Ya entre los facilitadores, han sido destacados: infraestructura institucional, imagen institucional y competencias en propiedad intelectual. Los resultados, además de identificar la existencia de intervinientes, permitieron corroborar estudios anteriores y crear, desde datos empíricos, una taxonomía de intervinientes, presentando su principal contribución a los estudios sobre transferencia tecnológica.

Palabras clave: Oficinas de Transferencia de Tecnología; Intervinientes en los Procesos de Transferencia Tecnológica; Transferencia de Tecnología.

\section{Introdução}

A partir do final do século XX o aumento da mobilidade dos trabalhadores do conhecimento e do volume de capital de risco privado corroeram as bases do modelo de inovação fechada e centrado nas atividades de pesquisa e desenvolvimento (P\&D) geradas internamente, levando as organizações a procurarem formas alternativas de inovar, culminando em um modelo de inovação aberta, onde as empresas buscam se apropriar de ideias externas para agregar valor para a organização (Chesbrough, 2003).

Aliado a isto, o fortalecimento de uma economia demanda que as descobertas tecnológicas oriundas da ciência sejam aplicadas ao setor produtivo e o Brasil historicamente não incentivava a criação de unidades de $P \& D$ em empresas, ficando a pesquisa restrita aos institutos de pesquisa que, por sua vez, não conseguiam interagir com o setor produtivo, resultando em um baixo índice de incorporação das tecnologias desenvolvidas nos produtos que circulam em nossos mercados (Matias-Pereira \& Kruglianskas, 2005).

Para mudar este cenário foi instituída a Lei 10.973/2004, que objetivou alcançar o desenvolvimento industrial brasileiro através do incentivo a realizações de pesquisas direcionadas ao ambiente produtivo (Silva, Kovaleski, \& Gaia, 2013). Esta legislação ampliou as possibilidades de interação entre instituições de pesquisa e o setor produtivo, levando a novos caminhos para que os resultados das pesquisas fossem convertidos em produtos de aplicação industrial (Muscio, 2010).

Dentro desse contexto e objetivando ampliar a compreensão sobre processos de interação entre instituições de pesquisa e empresas, este estudo busca investigar elementos que 

Thiago Borges Renault

influenciam processos de transferência de tecnologia (TT), tendo como unidade de análise o caso de uma instituição de ciência e tecnologia, a Fundação Oswaldo Cruz (Fiocruz).

Este artigo estrutura-se em cinco seções, iniciando-se com esta introdução ao tema. A seção dois apresenta o referencial teórico e, na sequência, a seção três expõe os procedimentos metodológicos adotados nesta pesquisa. A seção quatro aborda a apresentação e discussão dos resultados. Por fim, a seção cinco tece as considerações finais da pesquisa.

\section{Referencial teórico}

\subsection{Transferência de Tecnologia}

A TT envolve um movimento de tecnologias de uma organização para outra (Bozeman, 2000; Bozeman, Rimes, \& Youtie, 2015), sendo a base de conhecimento inerente ao processo (Bozeman, 2000). Desta forma a TT abrange um conjunto de atividades que viabilizam a transferência de conhecimentos, tecnologias, além de aspectos relativos a direitos de propriedade, convenções sociais e interesses econômicos (Brescia, Colombo, \& Landoni, 2016).

Para Bozeman et al. (2015) a TT envolve múltiplas partes interessadas que possuem objetivos diversos e, portanto, deve ser entendida dentro de um contexto particular, considerando tanto os agentes transferidor e receptor quanto o que e como está sendo transferido.

De acordo com Rogers, Takegami e Yin (2001) a conclusão da TT ocorre com a comercialização do produto e seu foco reside na interface entre a P\&D, normalmente executada por instituições governamentais, e a comercialização, desempenhada por empresas privadas.

Para que uma tecnologia seja transferida o agente transferidor deve utilizar mecanismos que viabilizem o processo de transferência (Berbegal-Mirabent, García, \& Ribeiro-Soriano, 2015; Bozeman et al., 2015; V. Kumar, U. Kumar, \& Persaud, 1999). Nesse sentido alguns estudos empíricos identificaram diversos mecanismos (Curi, Daraio, \& Llerena, 2012; Gils, Vissers, \& Wit, 2009; V. Kumar et al., 1999; Rogers et al., 2001; Takahashi, 2005), dentre os quais se destacam: contratos de $\mathrm{P} \& \mathrm{D}$; licenciamento; consórcios de pesquisa; fundos de pesquisa; parceria público-privada; spin-offs; vendas diretas; e incubadoras de empresas.

De acordo com a literatura a gestão da TT pode ser guiada pelas óticas da demanda e da oferta (Dias \& Porto, 2013, 2014). No primeiro caso uma organização detecta uma necessidade 
que não é suportada pela tecnologia disponível e, então, busca uma instituição que supra esta demanda (Deitos, 2002; U. Kumar, V. Kumar, Dutta, \& Fantazy, 2007). Posteriormente seleciona o mecanismo de transferência (Deitos, 2002; U. Kumar et al., 2007; Takahashi, 2005), realiza a negociação e efetiva a transferência, de modo que o receptor assimile a tecnologia e seja capaz de utilizá-la e aperfeiçoá-la (Deitos, 2002; Takahashi, 2005).

Já pela ótica da oferta a instituição desenvolve produtos, patenteia e divulga estes produtos objetivando que alguma instituição se interesse pela tecnologia (Rogers et al., 2001). Em seguida inicia a negociação, executa a transferência e aufere os resultados financeiros decorrentes dos direitos de propriedade (Rogers et al., 2001).

Brescia et al. (2016) enfatizam que os processos de TT tradicionalmente eram conduzidos pelos pesquisadores, porém as instituições de pesquisa criaram entidades estruturadas com o intuito de promover a gestão eficiente destes processos e o estímulo à comercialização dos resultados das pesquisas. São os Escritórios de Transferência de Tecnologia (ETT) ou Núcleos de Inovação Tecnológica (NIT) (Rosa \& Frega, 2017), que serão analisados na próxima seção.

\subsection{Escritórios de Transferência de Tecnologia}

No Brasil a figura do ETT passou a ser obrigatória a partir da Lei 10.973/2004, que determinou que institutos de pesquisa estruturassem um órgão interno responsável pela gestão da política institucional de inovação (Machado, Sartori, \& Crubellate, 2017).

Os ETT são responsáveis pelo estabelecimento de relações de cooperação entre instituições de pesquisa e o setor produtivo (Berbegal-Mirabent et al., 2015; Capart \& Sandelin, 2004), tendo como missão primordial alavancar a possibilidade de que descobertas das pesquisas sejam convertidas em produtos dos quais a sociedade possa usufruir (Capart \& Sandelin, 2004).

De acordo com Brescia et al. (2016) os ETT desenvolvem atividades sob o escopo de três áreas: suporte à propriedade intelectual (PI): atividades relativas aos direitos de PI e licenciamentos; suporte à pesquisa: atividades relativas à acordos legais e contratos de colaboração; e suporte à spin-offs: atividades que viabilizam a criação de novas empresas baseadas em pesquisa. 

Thiago Borges Renault

Dado o seu escopo de atuação os ETT têm se tornado estratégicos para a proteção, transferência e comercialização das descobertas oriundas de pesquisas ( $\mathrm{O}^{\prime}$ Kane, Mangematin, Geoghegan, \& Fitzgerald, 2015) ao atuar dando suporte tanto à realização de pesquisas quanto à divulgação e comercialização dos seus resultados (Siegel, Waldman, \& Link, 2003).

Como a atividade de comercialização é vista como algo pouco importante por grande parte dos pesquisadores (Derrick, 2015), os ETT adquirem papel fundamental na difusão da compreensão da importância da proteção intelectual das descobertas científicas (Siegel et al., 2003). Aliado a isto os ETT podem auxiliar pesquisadores a identificar aplicabilidade em suas pesquisas, bem como a conhecer mecanismos de proteção, transferência e comercialização (Desidério \& Zilber, 2014).

Desta forma os ETT devem acompanhar as pesquisas da instituição, objetivando viabilizar a transferência dos achados para o mercado (Desidério \& Zilber, 2014), atuando na proteção da PI (Lotufo, 2009; O'Kane et al., 2015) e interlocução com o mercado, via ações de divulgação, mediação de interesses (Lotufo, 2009; Muscio, 2010; O'Kane et al., 2015; Siegel et al., 2003) e formalização de institutos jurídicos adequados (Ribeiro, Rocha, \& Czelusniak, 2017).

As TT conduzidas por ETT são influenciadas por intervenientes que atuam sobre processos de cooperação e estes serão o objeto de análise da próxima seção.

\subsection{Intervenientes em Processos de Transferência de Tecnologia}

Processos de interação entre instituições de pesquisa e a indústria emergem a partir de uma pré-disposição para a cooperação, que tem como fonte motivadores que estimulem os atores a colaborar entre si (Noveli \& Segatto, 2012).

Noveli e Segatto (2012) elencam como motivadores para instituições de pesquisa: fontes adicionais de recursos; realização da função social institucional; aumento do conhecimento dos problemas existentes; incorporação de informações aos processos de ensino e pesquisa; divulgação da imagem da universidade; acesso a equipamentos mais modernos.

A realização da função social está associada à conversão do conhecimento desenvolvido internamente em aplicações que tragam benefício público (Closs, Ferreira, Sampaio, \& Perin, 2012; Noveli \& Segatto, 2012).

Em se tratando de recursos financeiros Closs et al. (2012), Rosa e Frega (2017) e Siegel, Waldman, Atwater e Link (2004) esclarecem que a possibilidade de ganhos financeiros atua 
como motivador ao auxiliar que pesquisadores consigam manter laboratórios e estudantes sob sua orientação.

Entretanto Baldini, Grimaldi e Sobrero (2007), Derrick (2015) e Rosa e Frega (2017) identificaram o reconhecimento científico e a promoção da carreira como principais motivadores que levam pesquisadores a se engajarem em processos de transferência.

Adicionalmente Noveli e Segatto (2012) esclarecem que as TT podem ser influenciadas por barreiras, que dificultam ou impedem a cooperação, ou por facilitadores, que atuam estimulando à colaboração.

De acordo com Garnica e Torkomian (2009) a estruturação dos ETT interfere sobre processos de transferência. Nesse sentido, a existência de escritórios especializados em PI e TT e o apoio dos NIT no monitoramento de oportunidades constituem elementos propulsionadores de TT (Garnica \& Torkomian, 2009). Já a existência de restrições para contratação e capacitação de pessoas (Torkomian, 2009), número reduzido ou insuficiente da equipe (Desidério \& Zilber, 2014; Dias \& Porto, 2013; Silva et al., 2013; Torkomian, 2009), elevado número de funcionários temporários (Dias \& Porto, 2013; Rosa \& Frega, 2017) e rotatividade (Desidério \& Zilber, 2014; Garnica \& Torkomian, 2009; Rosa \& Frega, 2017; Silva et al., 2013) representam barreiras.

As competências e habilidades dos ETT impactam sobre sua atuação (Olegário-da-Silva \& Segatto, 2017), representando um importante interveniente. Nesse sentido a existência de pessoal qualificado (Garnica \& Torkomian, 2009) e competências desenvolvidas por gestores de ETT (Rosa \& Frega, 2017) configuram facilitadores. Por outro lado, se caracterizam como barreiras: deficiências com o uso de língua inglesa, dificuldades de definição de royalties, pouco conhecimento sobre escalonamento de tecnologias, deficiências de gestão de projetos (Garnica \& Torkomian, 2009), dificuldade de valoração tecnológica (Garnica \& Torkomian, 2009; Lucena \& Sproesser, 2015), ausência de pessoal especializado na redação de patentes (Rosa \& Frega, 2017) e falta de habilidades para transferência e negociação (Desidério \& Zilber, 2014; Garnica \& Torkomian, 2009; Silva et al., 2013; Torkomian, 2009).

Dentro desse contexto Decter, Bennett e Leseure (2007), Desidério e Zilber (2014), G. C. Ferreira, Soria e Closs (2012), Garnica e Torkomian (2009) e Rosa e Frega (2017) apontam a capacidade de comunicação do ETT como elemento importante, se caracterizando como barreiras a falta de divulgação das atividades do ETT, disseminação da importância da proteção intelectual e prospecção de parceiros. 

Thiago Borges Renault

Conforme apontado por Olegário-da-Silva e Segatto (2017) as habilidades dos ETT podem ser incrementadas com a contratação de habilidades de empresas externas. Nesse sentido Dias e Porto (2013), Lucena e Sproesser (2015), Rosa e Frega (2017) apontam como facilitador a contratação de empresa especializada na redação de patentes.

Outro interveniente reside no fator cultural. Desta forma inventores envolvidos com o processo representam um facilitador (Garnica \& Torkomian, 2009). Por outro lado, a presença de diferenças culturais entre universidades e empresas, ausência de empreendedorismo (Decter et al., 2007), cultura de transferência pouco desenvolvida (Garnica \& Torkomian, 2009; Rosa \& Frega, 2017), publicação antes da proteção (Dias \& Porto, 2014), inexistência de cultura de proteção (Torkomian, 2009) e aversão à ideia de licenciar tecnologias (Rosa \& Frega, 2017; Thursby, Fuller, \& Thursby, 2009) configuram barreiras.

O desempenho dos ETT é influenciado pelas estruturas organizacionais e procuradorias jurídicas (Olegário-da-Silva \& Segatto, 2017). Dentro desse contexto, a ausência de interação entre departamentos (Desidério \& Zilber, 2014), número insuficiente de pessoal para o desenvolvimento de pesquisas (Garnica \& Torkomian, 2009) e ausência de política para avaliar o potencial de patenteabilidade de uma invenção (Dias \& Porto, 2013, 2014) foram apontados como barreiras. Com relação às procuradorias jurídicas Garnica e Torkomian (2009) esclarecem que o suporte jurídico representa um facilitador ao passo que Olegário-da-Silva e Segatto (2017) identificaram que a ação das procuradorias impacta negativamente sobre a agilidade dos ETT. Outros facilitadores relativos à estrutura organizacional incluem pesquisadores qualificados e laboratórios bem estruturados (Closs et al., 2012).

Diferenças de objetivos entre pesquisadores, engajados com pesquisas e publicações, e a indústria, que prioriza a comercialização, foram apontadas como intervenientes por Derrick (2015), uma vez que estas diferenças de percepção levam a conflitos que dificultam processos de transferência.

O fator tempo é apresentado como barreira por Closs et al. (2012) e Garnica e Torkomian (2009), estando atrelado à lentidão do processo jurídico-administrativo, excesso de burocracia e pouca flexibilidade. A burocracia também foi apontada como barreira por A. Ferreira, Amaral e Leopoldi (2013) e Rosa e Frega (2017).

Outro interveniente reside no aspecto financeiro. A carência de recursos financeiros para depósito e manutenção de patentes (Closs et al., 2012; Dias \& Porto, 2013) e ausência de financiamento para estratégias internacionais (Garnica \& Torkomian, 2009) representam obstáculos. Já como facilitadores foram apontadas tecnologias com elevada expectativa de 
retorno financeiro e expectativas de retribuição financeira para a instituição e o inventor (Garnica \& Torkomian, 2009).

Garnica e Torkomian (2009) apontam, também, a credibilidade como interveniente em processos de TT. Nesse sentido a elevada qualidade da tecnologia e credibilidade do inventor ou da instituição se mostram como facilitadores (Garnica \& Torkomian, 2009).

\section{Procedimentos metodológicos}

O presente estudo objetivou descortinar intervenientes dos processos de TT entre a Fiocruz e o setor produtivo. Para alcançar este objetivo foi adotada uma abordagem qualitativa, de modo a proporcionar uma compreensão detalhada do contexto envolvido (Creswell, 2014). A pesquisa foi conduzida de forma exploratório-descritiva, sendo caracterizada como descritiva ao externar especificidades do objeto de estudo (Vergara, 2015) e exploratória ao objetivar desenvolver a compreensão dos intervenientes dos processos de TT, desenvolvendo conceitos e ampliando o grau de proximidade com o fenômeno estudado (Gil, 2008).

O método utilizado para a condução da pesquisa foi o estudo de caso único (Yin, 2015), em razão da necessidade de compreensão em profundidade do caso e pelo caráter exploratório da pesquisa (Creswell, 2014).

A escolha da organização objeto de estudo justifica-se por sua representatividade no contexto da saúde pública nacional, devido ao impacto de suas pesquisas sobre as condições de saúde populacional. Aliado a isto, a Fiocruz teve o seu trabalho na seara da PI reconhecido internacionalmente ao ser indicada para a premiação Latin American Counsel Awards de 2017, na categoria de desempenho de times de PI (Global Counsel Awards [GCA], (2017).

Para contribuir para o rigor, a validade interna e a confiabilidade requeridos por estudos de caso, a coleta de dados foi efetuada de modo a permitir triangulação de dados (Yin, 2015), sendo obtidas evidências por meio de entrevistas semiestruturadas, pesquisa documental e observação direta.

As entrevistas foram realizadas com dez gestores do sistema de gestão tecnológica da Fiocruz, conforme apresentado pela Tabela 1. Os gestores foram selecionados de acordo com sua expertise em PI e vivência em processos de TT. Para a escolha dos sujeitos que atendessem a estes critérios, foram levadas em consideração indicações dos próprios entrevistados, por meio da técnica snowball, conforme recomendado por Creswell (2014). 
As entrevistas foram realizadas entre os meses de julho e setembro de 2017 e para elaboração do roteiro foram considerados fatores intervenientes em processos de TT identificados na literatura, de modo a detectar, a partir da percepção dos gestores, possíveis motivações que levaram ao desenvolvimento de tecnologias passíveis de transferência, bem como elementos que atuam como facilitadores ou barreiras à TT.

As observações foram realizadas em ciclos de debates sobre PI e TT da Fiocruz, ocorridos nos meses de setembro de 2016 e maio de 2017. A participação nestes eventos objetivou identificar informações sobre o contexto de PI da instituição, bem como obter dados sobre a atuação do sistema de gestão tecnológica organizacional.

\section{Tabela 1}

Entrevistas e observações efetuadas para o estudo de caso

\begin{tabular}{|c|c|c|c|}
\hline $\begin{array}{l}\text { Entrevista/ } \\
\text { observação }\end{array}$ & Função da unidade do NIT/evento & Critério de escolha & $\begin{array}{c}\text { Tempo } \\
\text { total } \\
\text { (minutos) } \\
\end{array}$ \\
\hline G-01 & Gestão da PI e da TT da Fiocruz & $\begin{array}{c}\text { Expertise PI e vivência em } \\
\text { TT }\end{array}$ & 82 \\
\hline G-02 & Pesquisas nas áreas biológica e biomédica & Vivência em TT & 37 \\
\hline G-03 & $\begin{array}{l}\text { Produção de medicamentos e insumos para a } \\
\text { saúde }\end{array}$ & Vivência em TT & 42 \\
\hline G-04 & $\begin{array}{l}\text { Produção de produtos biotecnológicos e insumos } \\
\text { para a saúde }\end{array}$ & Vivência em TT & 52 \\
\hline G-05 & $\begin{array}{c}\text { Assistência à saúde da mulher, da criança e do } \\
\text { adolescente }\end{array}$ & $\begin{array}{l}\text { Expertise em patentes e } \\
\text { vivência em TT }\end{array}$ & 56 \\
\hline G-06 & $\begin{array}{c}\text { Pesquisa e prestação de serviços para } \\
\text { diagnóstico de doenças infecciosas e genéticas e } \\
\text { controle de vetores }\end{array}$ & $\begin{array}{l}\text { Expertise em patentes e } \\
\text { vivência em TT }\end{array}$ & 72 \\
\hline G-07 e G-08 ${ }^{\mathrm{a}}$ & Preservação do patrimônio cultural da Fiocruz & Expertise em PI & 55 \\
\hline G-09 & Informação e comunicação em saúde & Expertise em PI & 53 \\
\hline G-10 & $\begin{array}{l}\text { Formação de recursos humanos nas áreas da } \\
\text { saúde coletiva }\end{array}$ & Expertise em PI & 30 \\
\hline O-01 & $\begin{array}{c}\text { Ciclo de Debates "Desafios da gestão da PI e da } \\
\text { inovação em uma instituição pública de } \\
\text { pesquisa" }\end{array}$ & $\begin{array}{c}\text { Informações sobre o } \\
\text { contexto nacional de PI e } \\
\text { sobre a atuação dos NIT }\end{array}$ & 360 \\
\hline O-02 & $\begin{array}{l}\text { Ciclo de Debates “A Conjuntura Política e o } \\
\text { Novo Marco Legal da Ciência, Tecnologia \& } \\
\text { Inovação - impactos para inovação em saúde" }\end{array}$ & $\begin{array}{c}\text { Informações sobre o } \\
\text { impacto novo marco legal } \\
\text { da inovação sobre as } \\
\text { unidades fabris da Fiocruz. }\end{array}$ & 60 \\
\hline
\end{tabular}

Fonte: Elaboração própria.

${ }^{\text {a }}$ Entrevista realizada simultaneamente com dois gestores.

As entrevistas totalizaram 479 minutos, sendo gravadas com a anuência dos entrevistados e integralmente transcritas. Já os dados oriundos das observações foram registrados em diário de campo. No que se refere à pesquisa documental, os dados foram coletados pela recuperação 
de notícias e documentos correlatos ao sistema de gestão tecnológico da Fiocruz, além da legislação que regula a TT.

Por fim, os dados foram submetidos à análise indutiva de Thomas (2006), técnica de análise de dados qualitativos guiada por objetivos específicos de avaliação, a partir da leitura detalhada dos dados brutos para gerar conceitos ou temas oriundos de sua interpretação. As categorias de análise foram estabelecidas tendo como parâmetros os intervenientes em processos de TT identificados na literatura. Os resultados encontrados são descritos e analisados na próxima seção.

\section{Apresentação e discussão dos resultados}

\subsection{Caracterização do Sistema de Gestão Tecnológica da Fiocruz}

A Coordenação de Gestão Tecnológica (Gestec) é a unidade responsável pela gestão tecnológica da Fiocruz, cuja missão reside em direcionar esforços para o aprimoramento da política de P\&D tecnológico da fundação e utilizar os mecanismos do Sistema Internacional de PI para que os resultados da pesquisa sejam incorporados pela sociedade (Coordenação de Gestão Tecnológica [Gestec], 2017a).

A atuação da Coordenação foi descentralizada a partir do ano de 2006, sendo instituída uma rede denominada Sistema Gestec-NIT, composta por dezenove NIT distribuídos pelas unidades institucionais, sob a coordenação da Gestec.

Os NIT estão subordinados às normatizações da Gestec e são responsáveis por interagir com pesquisadores, identificar oportunidades de inovação, orientar quanto a possibilidades de proteção, realizar buscas em bases de patentes e auxiliar nos processos de estabelecimento de parcerias (Gestec, 2017b).

\subsection{Gestão da Transferência Tecnológica na Fiocruz}

A Fiocruz atua sob as óticas da oferta (Rogers et al., 2001) e da demanda (Deitos, 2002; U. Kumar et al., 2007; Takahashi, 2005) de tecnologia.

Para ofertar tecnologias a Fiocruz necessita identificar instituições que possam se interessar pela tecnologia e a conexão com o mercado ocorre de forma proativa, pela prospecção 

Thiago Borges Renault

de parceiros, ou reativa, quando potenciais parceiros buscam a Fiocruz após consulta ao portal de portfólio de projetos.

Os mecanismos de TT utilizados em processos de oferta são o licenciamento de patentes ou direito autoral (Curi et al., 2012; Gils et al., 2009; V. Kumar et al., 1999; Rogers et al., 2001) e o desenvolvimento cooperativo (Curi et al., 2012; Gils et al., 2009; Rogers et al., 2001).

A escolha do mecanismo de TT é determinada pelo grau de maturação da tecnologia, sendo aquelas já concluídas e patenteadas transferidas via licenciamento e aquelas em estágio de protótipo ofertadas para parceiros com vistas ao desenvolvimento conjunto. Neste último caso os parceiros efetuam aprimoramentos ou a finalização da tecnologia e a patente é gerada em cotitularidade com a Fiocruz.

Após a negociação, os contratos são firmados com a área jurídica da Gestec e a tecnologia é transferida. O processo de transferência e pós-transferência envolve os inventores, que auxiliam os parceiros a absorver a tecnologia.

A absorção tecnológica (Deitos, 2002; U. Kumar et al., 2007; Takahashi, 2005) ocorre quando tecnologias são incorporadas e produzidas pelas unidades fabris da instituição. A absorção tecnológica normalmente acontece em decorrência das demandas do Ministério da Saúde para resolução de problemas reais de saúde pública, como nas campanhas de vacinação emergencial. Estas aquisições tecnológicas envolvem retribuição financeira ao detentor da patente.

\subsection{Intervenientes dos Processos de Transferência Tecnológica da Fiocruz}

Esta seção compreende uma síntese dos resultados empíricos referente aos intervenientes dos processos de TT da Fiocruz. Sua ordenação ocorreu a partir da frequência de aparição nos relatos dos entrevistados.

\subsubsection{Motivadores dos processos de transferência tecnológica da Fiocruz}

Assim como evidenciado pelos estudos de Closs et al. (2012) os dados empíricos evidenciaram que, em geral, o produto ou serviço oriundo das atividades de pesquisa da Fiocruz foi inesperado, decorrente do avanço da pesquisa. 
Dentro desse contexto foi identificado o reconhecimento científico como principal motivador para o desenvolvimento de pesquisas que resultem em transferências, tal como identificado por Baldini et al. (2007), Derrick (2015) e Rosa e Frega (2017).

Outra motivação evidenciada consiste no cumprimento da função social da instituição, corroborando os estudos de Closs et al. (2012) e Noveli e Segatto (2012). Neste contexto os pesquisadores buscam desenvolver pesquisas que possam contribuir para a resolução de adversidades na saúde pública.

A inclusão das patentes como métrica de produtividade dos pesquisadores levou a um aumento do interesse pelo patenteamento, porquanto amplia possibilidades de financiamento e manutenção de grupos de pesquisa, tal como motivador revelado pelas pesquisas de Closs et al. (2012), Rosa e Frega (2017) e Siegel et al. (2004).

\subsubsection{Barreiras dos processos de transferência tecnológica da Fiocruz}

As evidências que ilustram as barreiras identificadas pelos dados empíricos estão sumarizadas na Tabela 2.

A falta de direcionamento das pesquisas por produto evidencia a principal barreira à TT da Fiocruz: cultura não orientada para a TT. Os dados empíricos permitiram encontrar algumas evidências que vão ao encontro dos estudos de Decter et al. (2007), Dias e Porto (2014), Garnica e Torkomian (2009), Rosa e Frega (2017), Thursby et al. (2009) e Torkomian (2009). De acordo com os entrevistados, alguns pesquisadores são avessos à ideia de comercializar um produto gerado pela instituição, por acreditarem que proteger e comercializar tecnologias vão contra os preceitos de uma instituição pública. Este aspecto corrobora as pesquisas de Rosa e Frega (2017) e Thursby et al. (2009).

Outro obstáculo relacionado ao fator cultural reside na ansiedade pela publicação sem adequada proteção. Tal postura, embora possa dar renome ao pesquisador, faz com que o conhecimento caia em domínio público e outras empresas possam se apropriar da tecnologia e patenteá-la. Tal resultado converge com os achados de Dias e Porto (2014).

De maneira semelhante aos achados de Decter et al. (2007), Garnica e Torkomian (2009) e Torkomian (2009) os resultados empíricos revelaram que, em geral, o inventor não tem perfil empreendedor, o que faz com que oportunidades de inovação sejam perdidas porque os envolvidos desistem durante o processo de TT. 
Assim como identificado por Closs et al. (2012) e Garnica e Torkomian (2009) o fator tempo também foi apontado como barreira, porque o horizonte temporal de processos de TT é lento, burocrático e causa desmotivação ao inventor.

Também foi relatada como barreira a ausência de habilidades de valoração e negociação (Desidério \& Zilber, 2014; Garnica \& Torkomian, 2009; Lucena \& Sproesser, 2015; Silva et al., 2013; Torkomian, 2009), o que pode levar a instituição a firmar contratos que a prejudiquem. A elevada complexidade dos termos jurídicos empregados nos contratos tende a repelir empresas de menor porte. Além disso, quando uma tecnologia é desenvolvida por vários inventores torna-se difícil conciliar os diferentes interesses em processos de negociação. Nestes achados encontram-se algumas evidências congruentes aos estudos de Garnica e Torkomian (2009).

\section{Tabela 2}

Evidências relativas às barreiras em processos de TT da Fiocruz

\begin{tabular}{|c|c|}
\hline Barreiras & Extratos das entrevistas \\
\hline $\begin{array}{l}\text { Cultura não } \\
\text { orientada para a } \\
\text { TT }\end{array}$ & $\begin{array}{l}\text { "Ah... por exemplo, tem muitos pesquisadores que acham que por sermos uma } \\
\text { instituição pública, o dever deles é publicar a tecnologia que eles produzem e não } \\
\text { proteger isso pra... visando um licenciamento [...]" (G02). }\end{array}$ \\
\hline Fator tempo & $\begin{array}{l}\text { "[...] às vezes a gente começa uma negociação com uma empresa... [...] mas o tempo é } \\
\text { muito grande para eles né. [...] para eles vai se prolongando e aí no meio dessa trajetória } \\
\text { aí pode surgir outros... outros interesses e acaba se perdendo uma parceria que poderia } \\
\text { ser um sucesso no futuro" (G03). }\end{array}$ \\
\hline $\begin{array}{l}\text { Habilidades de } \\
\text { valoração e } \\
\text { negociação }\end{array}$ & $\begin{array}{l}\text { "Acho que existe também, talvez aí, um pouco de falta de preparo [...] então, de alguma } \\
\text { maneira a gente não consegue negociar bem aquilo que a gente produz e a gente vende } \\
\text { mal aquilo que a gente produz" (G09). }\end{array}$ \\
\hline $\begin{array}{l}\text { Dificuldade de } \\
\text { priorização de } \\
\text { projetos }\end{array}$ & $\begin{array}{l}\text { "[...] porque por muitas vezes você vê um projeto caminhando e não tem muito futuro, e } \\
\text { outro que deveria... teria um futuro e tá ali, ó [parado], então eu acho que essa é uma das } \\
\text { maiores dificuldades" (G03). }\end{array}$ \\
\hline $\begin{array}{l}\text { Dificuldade de } \\
\text { abdicar de } \\
\text { patentes sem } \\
\text { potencial de } \\
\text { mercado }\end{array}$ & $\begin{array}{l}\text { "E a gente não consegue abandonar as patentes sabia? [...] tem que ser uma decisão } \\
\text { institucional né, porque você está abrindo mão de um ativo. [...] como é que você faz } \\
\text { isso? O pesquisador fala não, não avancei a pesquisa, mas deixa aí a patente... às vezes } \\
\text { famílias internacionais tá? Geram um... assim... um custo caríssimo" (G01). }\end{array}$ \\
\hline $\begin{array}{l}\text { Recursos } \\
\text { financeiros }\end{array}$ & $\begin{array}{l}\text { "Muitas patentes a gente já perdeu porque não tinha um orçamento. Não tinha como } \\
\text { pagar... [...] já caiu patente por causa disso" (G01). }\end{array}$ \\
\hline $\begin{array}{l}\text { Estrutura de } \\
\text { governança } \\
\text { descentralizada }\end{array}$ & $\begin{array}{l}\text { "Cada unidade tem o seu NIT. Então o NIT está na unidade. A pessoa que está lá no } \\
\text { NIT, ela é um funcionário da unidade, então ela tem as férias lá, avaliação de } \\
\text { desempenho lá [...] a gente lida com isso, mas é uma complicação né" (G01). }\end{array}$ \\
\hline $\begin{array}{l}\text { Política de gestão } \\
\text { de pessoas }\end{array}$ & $\begin{array}{l}\text { "[...] na hora de fechar o perfil, fez um perfil com qualquer formação. [...] A gente } \\
\text { precisava de pessoas com determinados tipos de formação que seriam mais... é... como } \\
\text { eu falo isso? Mais, assim, a fim com esse trabalho né [...]" (G01). }\end{array}$ \\
\hline $\begin{array}{l}\text { Enfase em } \\
\text { patentes sem } \\
\text { considerar } \\
\text { transferências }\end{array}$ & $\begin{array}{l}\text { "Existe, só que a pontuação é pequena ainda. E de patenteamento... Isso eles se } \\
\text { interessam, porque eles querem melhorar o currículo... eu já ouvi isso de pesquisador... } \\
\text { agora na hora de negociar com parceiro não... "Não, isso eu não quero... Não me } \\
\text { interessa" (G02). }\end{array}$ \\
\hline $\begin{array}{l}\text { Imagem } \\
\text { institucional }\end{array}$ & $\begin{array}{l}\text { "teve um outro ta1 } \\
\text { tecnologias dela [pe }\end{array}$ \\
\hline
\end{tabular}




\begin{tabular}{ll}
\hline \multicolumn{1}{c}{ Barreiras } & \multicolumn{1}{c}{ Extratos das entrevistas } \\
\hline & $\begin{array}{l}\text { estrutura nenhuma... e... esse era o típico caso que ele queria uma parceria de nome, } \\
\text { mas dificilmente conseguiria efetivar alguma coisa" (G02). }\end{array}$ \\
\hline $\begin{array}{l}\text { Diferenças de } \\
\text { percepção e } \\
\text { objetivos entre } \\
\text { pesquisadores e } \\
\text { empresários }\end{array}$ & $\begin{array}{l}\text { "São mundos [pesquisa e produção] que precisam conversar. Há a necessidade, mas } \\
\text { produção. [...] se você não tem esse conhecimento das possibilidades e das necessidades } \\
\text { de adaptações, isso geralmente barra, quando chega na parte da produção não é viável” }\end{array}$ \\
\hline $\begin{array}{l}\text { Contexto } \\
\text { econômico } \\
\text { desfavorável }\end{array}$ & $\begin{array}{l}\text { a mos...] a empresa teoricamente faz P\&D, e ele disse olha, cortei o meu P\&D, eu vou para } \\
\text { dizer, a gente não tem um cenário na prática pró-inovação, quando você vê o contexto, }\end{array}$ \\
\hline
\end{tabular}

Fonte: Elaboração própria.

Embora o relato dos entrevistados aponte que a Fiocruz procura patentear somente tecnologias com potencial de mercado, não há espelhamento desta postura para a inicialização e manutenção de projetos. Esta dificuldade de priorização de projetos prejudica o direcionamento de recursos apenas para projetos que tenham aplicabilidade real. Tal achado converge com os estudos de Dias e Porto (2013, 2014).

Também foi verificada a existência de uma grande dificuldade de a instituição abdicar de patentes sem potencial de mercado, que não geram contrapartida para a sociedade. Esta barreira não foi verificada na literatura e ocorre normalmente porque o pesquisador não avançou na condução da pesquisa.

O comprometimento de recursos com projetos ou patentes sem potencial de mercado contribuiu para o surgimento da barreira de carência de recursos financeiros, razão majoritária para a perda de patentes ao dificultar o pagamento dos custos de manutenção. Esta barreira foi identificada por Closs et al. (2012) e Dias e Porto (2013).

Outra barreira não identificada na literatura refere-se à governança do sistema de gestão tecnológica institucional, onde os NIT são formados por pessoas não subordinadas à Gestec. Tal característica dificulta a gestão da rede, demandando elevado potencial de convencimento por parte da Gestec.

De maneira semelhante aos achados de Torkomian (2009), os entrevistados apontaram entraves relacionados à política de gestão de pessoas. Nesse sentido foram mencionadas a inexistência de orçamento formal para capacitação e deficiências na seleção por concurso público, que não obteve êxito na captação de profissionais com perfil adequado para atuar na área. 

Thiago Borges Renault

O motivador relacionado à adoção de patentes com métrica de produtividade releva outra barreira: ênfase em patentes sem considerar possíveis transferências. Os pesquisadores têm buscado patentear, mas visando apenas o incremento do seu currículo, permanecendo avessos ao ingresso na seara da TT. Estes dados confirmam que a expectativa de ganhos financeiros eleva o interesse pelo patenteamento, porém a ausência de perfil empreendedor e cultura de TT impactam negativamente sobre a TT (Decter et al., 2007; Garnica \& Torkomian, 2009).

Outro interveniente identificado refere-se à imagem institucional, que representa uma barreira quando instituições buscam se associar a uma renomada instituição, mas sem ter intenção real de absorver a tecnologia, retardando a efetivação de transferências. Este aspecto vai de encontro aos achados de Garnica e Torkomian (2009), que identificaram a credibilidade somente enquanto facilitador.

Diferenças de percepção e objetivos entre pesquisadores e empresários também foram apontadas enquanto barreiras, gerando dificuldades de comunicação e embates que podem retardar ou inviabilizar processos de transferência. Estes pontos ratificam as barreiras identificadas por Decter et al. (2007) e Derrick (2015).

O contexto econômico desfavorável também foi apontado como barreira porque, para contornar a crise econômica, as empresas têm cortado gastos com $\mathrm{P} \& \mathrm{D}$, dificultando o estabelecimento de parcerias. Este aspecto também não foi verificado na literatura sobre o tema.

\subsubsection{Facilitadores dos processos de transferência tecnológica da Fiocruz}

As evidências que ilustram os facilitadores descortinados pelos dados empíricos estão sumarizadas na Tabela 3.

A infraestrutura institucional foi apontada como principal facilitador. A Fiocruz possui infraestrutura para o desenvolvimento de pesquisas, protótipos e produção. Desta forma o desempenho dos processos de TT é impactado positivamente pela infraestrutura de pesquisa e produção, assim como identificado por Closs et al. (2012).

Embora a imagem institucional tenha sido apontada como barreira, a credibilidade da Fiocruz tende a atrair instituições interessadas em estabelecer parcerias, que podem se converter em transferências exitosas. Desta forma a imagem institucional também se caracteriza como facilitador, tal como identificado por Garnica e Torkomian (2009).

A despeito da barreira cultural a promoção de ações de estímulo a criação de uma cultura de proteção foi apontada como um facilitador. A instituição vem ofertando disciplina 
de PI em seus cursos de pós-graduação, objetivando aumentar a conscientização sobre a importância da proteção. Outras medidas incluem o estímulo à codificação de resumos e metodologias de artigos e a adoção de bancas de pós-graduação fechadas. Tais ações são importantes para o aumento das atividades de TT, conforme apontado por Decter et al. (2007), Desidério e Zilber (2014), G. C. Ferreira et al. (2012), Garnica e Torkomian (2009) e Rosa e Frega (2017).

A proximidade da Gestec com o Instituto Nacional da Propriedade Industrial (INPI) viabilizou o desenvolvimento de competências relativas à gestão da PI, importante competência de um ETT (Olegário-da-Silva \& Segatto, 2017) e que facilita as atividades de transferência (Rosa \& Frega, 2017).

\section{Tabela 3}

Evidências relativas aos facilitadores dos processos de TT da Fiocruz

\begin{tabular}{|c|c|}
\hline Facilitadores & Extratos das entrevistas \\
\hline $\begin{array}{l}\text { Infraestrutura } \\
\text { institucional }\end{array}$ & $\begin{array}{l}\text { "[...] a gente tem um sistema estruturado, tem um acompanhamento durante o antes, o } \\
\text { durante e o depois, que eu digo assim, a pesquisa básica, a pesquisa tecnológica, de P\&D, } \\
\text { fazer uma pesquisa e desenvolvimento [...] eu acho que hoje a gente tem um sistema que } \\
\text { facilite que a transferência ocorra" (G05). }\end{array}$ \\
\hline $\begin{array}{l}\text { Imagem } \\
\text { Institucional }\end{array}$ & $\begin{array}{l}\text { "A Fiocruz é uma instituição que tem um nome, é reconhecida nacionalmente e } \\
\text { internacionalmente, então acho que os nossos pesquisadores possuem uma certa } \\
\text { facilidade de chegar em uma outra instituição, de conversar, de buscar até uma parceria, } \\
\text { porque é uma instituição confiável" (G03). }\end{array}$ \\
\hline $\begin{array}{l}\text { Ações de estímulo } \\
\text { à cultura de } \\
\text { proteção }\end{array}$ & $\begin{array}{l}\text { "[...] a gente investe mesmo aqui na disciplina de propriedade intelectual. Tem uma } \\
\text { disciplina aqui na pós-graduação [...] porque a nossa intenção é formar uma nova geração } \\
\text { que entenda isso e não venha com esses preconceitos, porque é muito difícil você tirar } \\
\text { isso da cabeça de um pesquisador já formado, já mais antigo, sabe..." (G02). }\end{array}$ \\
\hline $\begin{array}{l}\text { Competências } \\
\text { relativas à gestão } \\
\text { da } \mathbf{P I}\end{array}$ & $\begin{array}{l}\text { "A gente também morou um tempão no INPI para aprender todos os processos } \\
\text { relacionados a depósitos de patente e transferência de tecnologia. [...] então a gente foi } \\
\text { conseguindo assim adquirir as competências" (G01). }\end{array}$ \\
\hline $\begin{array}{l}\text { Alinhamento da } \\
\text { Gestec com outras } \\
\text { unidades }\end{array}$ & \multirow{2}{*}{$\begin{array}{l}\text { "[...] o pessoal que cuida dos pagamentos, eles têm toda a noção do processo, } \\
\text { procuradoria também tem toda a noção do processo... ao longo do tempo a gente } \\
\text { construiu um entendimento muito bom com eles, e eles fazem tudo o que você pode } \\
\text { imaginar para não correr o risco de perder um ativo intangível da instituição que é tão } \\
\text { difícil de você conseguir né... [...]" (G01). }\end{array}$} \\
\hline $\begin{array}{l}\text { Interação com a } \\
\text { Procuradoria }\end{array}$ & \\
\hline
\end{tabular}

Fonte: Elaboração própria.

Foi relatado o alinhamento da Gestec com outras unidades da Fiocruz como um facilitador. Nesse sentido existe uma proximidade com a área que faz o pagamento das patentes, minimizando as chances de perda de patentes. Desta forma, diferentemente dos achados de Desidério e Zilber (2014), a interação entre os departamentos atua como facilitador.

Os processos de TT da Fiocruz também são impactados pelas competências de sua área jurídica, assim como identificado por Olegário-da-Silva e Segatto (2017). Os entrevistados 
relataram haver uma boa interação com a Procuradoria da Fiocruz e o respaldo jurídico facilita a condução dos processos de TT, convergindo com os achados de Garnica e Torkomian (2009).

\section{Considerações finais}

Esta pesquisa objetivou investigar os intervenientes dos processos de transferência de tecnologia da Fiocruz a partir da perspectiva dos gestores do sistema de gestão tecnológica institucional. A caracterização do estudo dos processos de TT do caso Fiocruz permitiu identificar como motivadores o reconhecimento científico, o cumprimento da função social da instituição e a possibilidade de financiamento e manutenção de grupos de pesquisa, consoante motivadores identificados em estudos anteriores (Baldini et al., 2007; Closs et al., 2012; Derrick, 2015; Noveli \& Segatto, 2012; Rosa \& Frega, 2017).

Verificou-se, também, a existência de facilitadores, dentre os quais se sobressaem a infraestrutura organizacional e as competências relativas à PI, corroborando os estudos de Closs et al. (2012), Olegário-da-Silva e Segatto (2017) e Rosa e Frega (2017).

A Fiocruz é uma instituição que integra ensino e pesquisa, dispõe de laboratórios, unidades fabris e presta atendimento assistencial. Desta forma o pesquisador dispõe de públicoalvo, estrutura e pessoal capacitado para conduzir pesquisas e gerenciar seus resultados. Já o estabelecimento de uma proximidade com o INPI permitiu que os gestores desenvolvessem competências no que tange a buscas de anterioridade, redação e depósito de patentes.

Outros facilitadores incluem a imagem institucional, alinhamento da Gestec com outras unidades da Fiocruz, interação com a procuradoria jurídica e promoção de ações de estímulo à cultura de proteção. Tais achados apresentam algumas evidências que convergem com as pesquisas de Decter et al. (2007), Desidério e Zilber (2014), G. C. Ferreira et al. (2012), Garnica e Torkomian (2009), Olegário-da-Silva e Segatto (2017) e Rosa e Frega (2017).

No âmbito das barreiras foram identificados: cultura não orientada para a TT, fator tempo, ausência de habilidades de valoração e negociação, dificuldade de priorização de projetos, carência de recursos financeiros, política de gestão de pessoas, ênfase em patentes sem considerar transferências, imagem institucional e diferenças de percepção entre pesquisadores e empresários. Em tais achados foram encontradas algumas evidências congruentes às pesquisas de Closs et al. (2012), Decter et al. (2007), Derrick (2015), Desidério e Zilber (2014), Dias e 
Porto (2013, 2014), Garnica e Torkomian (2009), Lucena e Sproesser (2015), Rosa e Frega (2017), Silva et al. (2013), Thursby et al. (2009) e Torkomian (2009).

Cabe salientar que foram identificadas barreiras não verificadas na literatura, caracterizadas pela dificuldade de abdicar de patentes sem potencial de mercado, governança do sistema descentralizado de gestão tecnológica e contexto econômico desfavorável. Tais barreiras estão atreladas à aspectos da política de gestão da inovação adotada pela Fiocruz e à crise econômica atualmente vivenciada pelo país.

Além de identificar a existência de intervenientes, este estudo permitiu descrever o processo de gestão da TT da Fiocruz e criar, a partir de dados empíricos, uma taxonomia de intervenientes, acrescentando sua principal contribuição aos estudos sobre TT. O estudo permitiu, também, corroborar pesquisas anteriores sobre intervenientes de processos de TT.

No que diz respeito às limitações do presente estudo, cabe salientar a impossibilidade de generalizações a partir de um estudo de caso único. Entretanto, conforme apontado por Yin (2015), é possível haver a generalização analítica a partir da lógica de replicação de diversos casos e, sob esta ótica, propõe-se, a título de estudos futuros, investigar o tema em outras instituições públicas de ciência e tecnologia nacionais, para que os resultados sejam consolidados e geradas proposições teóricas aplicáveis a outros contextos.

Por fim, sugere-se a realização de estudos que avaliem intervenientes sob a perspectiva de outras partes envolvidas em processos de transferência, como inventores ou parceiros, de modo a confrontar com os achados identificados a partir da perspectiva dos gestores e validálos.

\section{Referências}

Baldini, N., Grimaldi, R., \& Sobrero, M. (2007). To patent or no to patent? A survey of Italian inventors on motivations, incentives and obstacles to university patenting. Scientometrics, 70(2), 333-354. doi: 10.1007/s11192-007-0206-5

Berbegal-Mirabent, J., García, J. L. S., \& Ribeiro-Soriano, D. E. (2015). University-industry partnerships for the provision of R\&D services. Journal of Business Research, 68(7), 14071413. doi: 10.1016/j.jbusres.2015.01.023

Bozeman, B. (2000). Technology transfer and public policy: a review of research and theory. Research Policy, 29(4/5), 627-655. doi: 10.1016/S0048-7333(99)00093-1 

Thiago Borges Renault

Bozeman, B., Rimes, H., \& Youtie, J. (2015). The evolving state-of-the-art in technology transfer research: revisiting the contingent effectiveness model. Research Policy, 44(1), 3449. doi: 10.1016/j.respol.2014.06.008

Brescia, F., Colombo, G., \& Landoni, P. (2016). Organizational structures of knowledge transfer offices: an analysis of the world's top-ranked universities. The Journal of Technology Transfer, 41(1), 132-151. doi: 10.1007/s10961-014-9384-5

Capart, G., \& Sandelin, J. (2004). Models of, and missions for, transfer offices from public research organizations. Recuperado de http://otl.stanford.edu/documents/JSMissionsModelsPaper-1.pdf

Chesbrough, H. W. (2003). The era of open innovation. MIT Sloan Management Review, 44(3), $35-41$.

Closs, L., Ferreira, G., Sampaio, C., \& Perin, M. (2012). Intervenientes na transferência de tecnologia universidade-empresa: o caso PUCRS. Revista de Administração Contemporânea, 16(1), 61-78. doi: 10.1590/S1415-65552012000100005

Coordenação de Gestão Tecnológica. (2017a). O Sistema Gestec-NIT. Recuperado em 03 abril, 2017, de http://www.fiocruz.br/vppis/gestec/sistema.php

Coordenação de Gestão Tecnológica. (2017b). Quem somos. Recuperado em 03 abril, 2017, de http://www.fiocruz.br/vppis/gestec/quemsomos.php

Creswell, J. W. (2014). Investigação qualitativa e projeto de pesquisa: escolhendo entre cinco abordagens (3a ed.). Porto Alegre: Penso.

Curi, C., Daraio, C., \& Llerena, P. (2012). University technology transfer: how (in)efficient are French universities? Cambridge Journal of Economics, 36(3), 629-654. doi: 10.1093/cje/bes020

Decter, M., Bennett, D., \& Leseure, M. (2007). University to business technology transferUK and USA comparisons. Technovation, 27(3), 145-155. doi: 10.1016/j.technovation.2006.02.001

Deitos, M. L. M. S. (2002). A gestão da tecnologia em pequenas e médias empresas: fatores limitantes e formas de superação. Cascavel: Edunioeste.

Derrick, G. E. (2015). Integration versus separation: structure and strategies of the technology transfer office (TTO) in medical research organizations. The Journal of Technology Transfer, 40(1), 105-122. doi: 10.1007/s10961-014-9343-1

Desidério, P. H. M., \& Zilber, M. A. (2014). Barreiras no processo de transferência tecnológica entre agências de inovação e empresas: observações em universidades públicas e privadas. Revista Gestão \& Tecnologia, 14(2), 99-124. doi: 10.20397/2177-6652/2014.v14i2.650

Dias, A. A., \& Porto, G. S. (2013). Gestão de transferência de tecnologia na Inova Unicamp. Revista de Administração Contemporânea, 17(3), 263-284. doi: 10.1590/S141565552013000300002. 
Dias, A. A., \& Porto, G. S. (2014). Como a USP transfere tecnologia? Organizações \& Sociedade, 21(70), 489-508. doi: S1984-92302014000300008

Ferreira, A., Amaral, M., \& Leopoldi, M. A. (2013). Análise da interação universidade-empresa sob a perspectiva do corpo docente: um estudo de caso em uma universidade pública. Revista de Administração, Contabilidade e Economia, 12(2), 677-708. Recuperado de http://www.spell.org.br/documentos/download/37576.

Ferreira, G. C., Soria, A. F., \& Closs, L. (2012). Gestão da interação Universidade-Empresa: o caso PUCRS. Sociedade e Estado, 27(1), 79-94. doi: 10.1590/S010269922012000100006

Garnica, L. A., \& Torkomian, A. L. V. (2009). Gestão de tecnologia em universidades: uma análise do patenteamento e dos fatores de dificuldade e de apoio à transferência de tecnologia no Estado de São Paulo. Gestão \& Produção, 16(4), 624-638. doi: 10.1590/S0104530X2009000400011

Gil, A. C. (2008). Métodos e técnicas de pesquisa social (6a ed.). São Paulo: Atlas.

Gils, M. van, Vissers, G., \& Wit, J. de (2009). Selecting the right channel for knowledge transfer between industry and science: consider the R\&D-activity. European Journal of Innovation Management, 12(4), 492-511. doi: 10.1108/14601060910996936

Global Counsel Awards. (2017). Latin American Counsel Awards - Shortlist 2017. Recuperado em 20 dezembro, 2017, de https://www.globalcounselawards.com/lca/shortlist/2017-2-2

Kumar, U., Kumar, V., Dutta, S., \& Fantazy, K. (2007). State sponsored large scale technology transfer projects in a developing country context. The Journal of Technology Transfer, 32(6), 629-644. doi: 10.1007/s10961-006-8880-7

Kumar, V., Kumar, U., \& Persaud, A. (1999). Building technological capability through importing technology: the case of Indonesian manufacturing industry. The Journal of Technology Transfer, 24(1), 81-96. doi: 10.1023/A:1007728921126

Lotufo, R. A. (2009). A institucionalização de Núcleos de Inovação Tecnológica e a experiência da inova Unicamp. In M. Santos, P. Toledo, \& R. Lotufo (Eds.), Transferência de tecnologia: estratégias para a estruturação e gestão de núcleos de inovação tecnológica (pp. 41-73). Campinas: Komedi.

Lucena, R. M., \& Sproesser, R. L. (2015). Análise da Gestão de Licenciamento de Patentes: estudo Multicasos de Instituições Federais de Ensino Superior. Revista de Administração e Inovação, 12(3), 28-55. doi: 10.11606/rai.v12i3.100934

Machado, H. P. V., Sartori, R., \& Crubellate, J. M. (2017). Institucionalização de Núcleos de Inovação Tecnológica em Instituições de Ciência e Tecnologia da Região Sul do Brasil. REAd. Revista Eletrônica de Administração (Porto Alegre), 23(3), 5-31. doi: 10.1590/14132311.177.67190

Matias-Pereira, J., \& Kruglianskas, I. (2005). Gestão de inovação: a lei de inovação tecnológica como ferramenta de apoio às políticas industrial e tecnológica do Brasil. RAE Revista de Administração de Empresas - eletrônica, 4(2). doi: 10.1590/S1676-56482005000200003 

Thiago Borges Renault

Muscio, A. (2010). What drives the university use of technology transfer offices? Evidences from Italy. Journal of Technology Transfer, 35(2), 181-202. doi: 10.1007/s10961-009-91217

Noveli, M., \& Segatto, A. P. (2012). Processo de cooperação universidade-empresa para a inovação tecnológica em um parque tecnológico: evidências empíricas e proposição de um modelo conceitual. Revista de Administração e Inovação, 9(1), 81-105. doi: 10.5773/rai.v1i1.610

O'Kane, C., Mangematin, V., Geoghegan, W., \& Fitzgerald, C. (2015). University technology transfer offices: the search for identity to build legitimacy. Research Policy, 44(2), 421-437. doi: 10.1016/j.respol.2014.08.003

Olegário-da-Silva, A. C., \& Segatto, A. P. (2017, outubro). Competências organizacionais dos Escritórios de Transferência de Tecnologia: evidências do contexto brasileiro. Anais do Encontro Nacional da Associação Nacional de Pós-Graduação e Pesquisa em Administração, São Paulo, SP, Brasil, 41.

Ribeiro, M. C. P., Rocha, W. F., Jr., \& Czelusniak, V. A. (2017). Mecanismos jurídicos e econômicos para a transferência de tecnologia: um estudo de caso. Revista Direito $G V$, 13(1), pp. 49-68. doi: 10.1590/2317-6172201703

Rogers, E. M., Takegami, S., \& Yin, J. (2001). Lessons learned about technology transfer. Technovation, 21(4), 253-261. doi:10.1016/S0166-4972(00)00039-0

Rosa, R. A., \& Frega, J. R. (2017). Intervenientes do Processo de Transferência Tecnológica em uma Universidade Pública. Revista de Administração Contemporânea, 21(4), 435-457. doi: 10.1590/1982-7849rac2017160097

Siegel, D. S., Waldman, D. A., Atwater, L. E., \& Link, A. N. (2004). Toward a model of the effective transfer of scientific knowledge from academicians to practitioners: qualitative evidence from the commercialization of university technologies. Journal of Engineering and Technology Management, 21(1-2), 115-142. doi: 10.1016/j.jengtecman.2003.12.006

Siegel, D. S., Waldman, D., \& Link, A. (2003). Assessing the impact of organizational practices on the relative productivity of university technology transfer offices: an exploratory study. Research Policy, 32(1), 27-48. doi: 10.1016/S0048-7333(01)00196-2

Silva, L. C. S., Kovaleski, J. L., \& Gaia, S. (2013). Gestão do conhecimento organizacional visando à transferência de tecnologia: os desafios enfrentados pelo NIT da Universidade Estadual de Santa Cruz. Produção Online, 13(2), 677-702. Doi: 10.14488/16761901.v13i2.1314

Takahashi, V. P. (2005). Transferência de conhecimento tecnológico: estudo de múltiplos casos na indústria farmacêutica. Gestão \& Produção, 12(2), 255-269. doi: 10.1590/S0104530X2005000200009

Thomas, D. R. (2006). A general inductive approach for analyzing qualitative evaluation data. American Journal of Evaluation, 27(2), 237-246. doi: 10.1177/1098214005283748 
Thursby, J., Fuller, A., \& Thursby, M. (2009). US faculty patenting: inside and outside the university. Research Policy, 38(1), 14-25. doi: 10.1016/j.respol.2008.09.004

Torkomian, A. L. V. (2009). Panorama dos núcleos de inovação tecnológica no Brasil. In M. Santos, P. Toledo, \& R. Lotufo (Eds.), Transferência de tecnologia: estratégias para a estruturação e gestão de núcleos de inovação tecnológica (pp. 21-38). Campinas: Komedi.

Vergara, S. C. (2015). Métodos de pesquisa em Administração (6a ed.). São Paulo: Atlas.

Yin, R. K. . (2015). Estudo de caso: planejamento e métodos (5a ed.). Porto Alegre: Bookman. 